\title{
Empowering SMEs to make better decisions with Business Intelligence: A Case Study
}

\author{
Raghavendra Raj ${ }^{1}$, Shun Ha Sylvia Wong ${ }^{2}$ and Anthony J. Beaumont ${ }^{2}$ \\ ${ }^{1}$ AGGORA Group, North Moons Moat, Redditch B98 7UX, U.K.. \\ raghavendra.rajeaggora.com \\ ${ }^{2}$ School of Engineering and Applied Science, Aston University, Aston Triangle, Birmingham \\ B4 7ET, U.K. \\ \{s.h.s.wong, a.j.beaumont\} easton.ac.uk
}

\begin{abstract}
With the advance of Business Information Systems (BIS), irrespective of the size, companies have adopted an approach to electronic data collection and management for two decades. The advancement in technology means they have in their possessions large volumes of historical data. Large organizations have cached on this and use a range of tools and techniques to leverage the usefulness of this information to make more informed business decisions. For most small and medium-sized enterprises (SMEs), however, such data typically sits in an archive without being utilized. While SMEs appreciate the need for utilizing historical data to make more informed business decisions, they often lack the technical knowhow and funding to embrace an effective BI solution. In this paper, drawing from our experience in implementing a BI solution for a UK SME we discuss some potential tools and strategies that could help SMEs overcome these challenges so as to reap the benefits of adopting an effective BI solution.
\end{abstract}

Keywords: Business Intelligence, Data Warehouse, Microsoft BI, SME.

\section{Introduction}

Information Technology (IT) has become an essential for businesses of any size for over three decades. For most businesses, this has facilitated the collection of a vast amount of business transaction data. While such data are important to support smooth operations of the company at the time of its creation, once the respective business transactions have been dealt with, they are often being archived away and are unlikely to ever be revisited. However, such historic data, when analyzed appropriately, can provide important clues to discover new business opportunities and to improve the company's business processes. The ability to efficiently manage, access and analyze large volumes of company historic data means that business decision making is more informed and business trends and risks can be more easily identified.

Business Intelligence (BI) rediscovers the usefulness of existing business information. It equips managers and decision makers with important information to 
perform business analyses that are needed for making key business decisions. BI has been a core strategy for growth in large corporations for more than twenty years. The driver being the relative ease by which large amounts of business data can be collected from various sources. With the rapid changes in business climate and conditions, even small and medium-sized enterprises (SMEs) have increasingly look to adopting $\mathrm{BI}$ in supporting their business decision making process. However, when looking to adopting a BI solution, many SMEs encounter issues such as a general lack of technical expertise to convert transaction data into business information and the general lack of funding to invest in a suitable BI solution. Furthermore, the lack of understanding of the benefits of BI also makes SMEs reluctant to invest in the adoption of new BI solutions. Without a BI solution, the decision-making in SMEs is reliant on the results from various Information and Communications Technology (ICT) tools incorporated into the company's infrastructure. These are unlikely to be designed to perform business analysis [26] and their use for that function may adversely affect their use to support live business needs and the results may be restricted by the structure, distribution and the correctness of the data, leading to suboptimal business decisions being made. Simply possessing the data does not mean you can extract business intelligence from it.

Business Intelligence is not a novel concept. This term was first put forward by Luhn [15] and was reintroduced by Howard Dresner in 1989 [2]. As Negash and Gray [24] explained, BI systems are specialist IT systems which "combine data gathering, data storage, and knowledge management with analytical tools to present complex and competitive information to planners and decision-makers". Such systems typically analyze data from a centralized data repository which hosts business and company data aggregated from various sources. Due to the advancement of BI tools, the adoption of BI has grown significantly since then. Furthermore, the readiness of companies to adopt new strategies to stay ahead of their game also pushes up the demand for high quality business intelligence. This has led to more concerted efforts being placed in developing new BI technologies. Recent advances in technologies such as Decision Support Systems (DSS), Executive Information Systems (EIS), Data Warehouse (DW), Online Analytical Processing (OLAP) and Data Mining have also improved the capabilities of BI systems and have played a significant role in raising awareness, and also in increasing popularity, of Business Intelligence in the business sector.

With the increased popularity of BI amongst businesses, proven benefits of adopting BI in businesses have emerged. For example, Watson and Wixom [29] reported that implementing BI solutions could lead to faster and cheaper information retrieval, thus helping organisations to achieve their business goals. Howson [11] noted that BI helped employees in all divisions to interact with, and analyze, business data in order to facilitate a more informed business management process. This has led to an increase in company efficiency, the ability to identify new business opportunities and the ability to facilitate operation reengineering. A survey of more than 2,300 CIOs published by Gartner in 2012 [6] revealed that BI was ranked the top 
technology priority by the participating companies as BI enabled them to create new capabilities. As summarized by Chugh and Grandhi [3], the key benefits of implementing BI solutions in an enterprise includes:

- Equipping the company with the ability to analyze data from multiple sources and using different dimensions;

- Enabling managers to make informed business decisions through identifying important patterns of behaviour captured in the data;

- Improving accuracy in predictions;

- Helping the company to identify root causes of problems so as to improve operational efficiency.

A good range of businesses have been utilizing BI to assist in making key business decisions for many years, e.g. banking, financial services, health service, IT companies, insurance companies, manufacturing industry, etc. A majority of large organizations are already drawing benefits from using BI solutions. For many of them, BI has become one of the major strategies to maintain a competitive edge. Furthermore, contrary to what it may seem, BI is not just for top-level management such as Managing Directors. When appropriately implemented, BI can empower a wide range of business decision-making processes. As was reported by Horakova and Skalska [10], BI is not restricted to top-level management, different company departments and business units use some kind of BI tools and the main users of BI include managers from sales, marketing, purchasing, accrual, finance, accounting, human resource and IT. As was also noted in their preliminary study, Raj et al. [26] reported that BI solutions has potential to inform business decisions across different layers of managements within an SME, it also helps to highlight operational issues. In light of the progress made since the work was presented by Raj et al. [26], this paper reports the new findings and recorded project benefits while giving more details on the BI implementation approach adopted and the lessons learnt.

The rest of this paper is divided into three parts. The first part reviews a range of BI solutions and, in light of the current adoption ratio of BI solutions amongst SMEs, we discuss some challenges faced by SMEs when considering to adopt BI solutions. In the second part of this paper, we present a case study on BI adoption and implementation by a UK SME, and the benefits attained by the SME as a result of the BI solution implementation. The last part of this paper explains the lessons learnt and gives an insight on some of the substantial future work.

\section{BI Solutions}

BI solutions typically analyze data from a centralized data repository which hosts business and company data aggregated from various sources within the organization 
and comprise multiple technological components. To generate business intelligence, data from business transactions and other relevant company processes needs to undergo an Extract-Transform-Load (ETL) process. This process cleanses, transforms and restructures business data and stores it in a data warehouse or a data mart. Data warehouses and data marts are essentially databases specially designed for promoting data analysis and knowledge discovery. How to analyse the data is dependent on the needs of the company and hence varies from business to business. One common area of data analyses is driven by the company's Key Performance Indicators (KPIs). To assist in knowledge discovery, data visualization tools are often used to present the results of data analyses to BI users. These results may be presented as standard KPI reports generated from regular queries or as on-demand reports that are generated for informing specific business decisions.

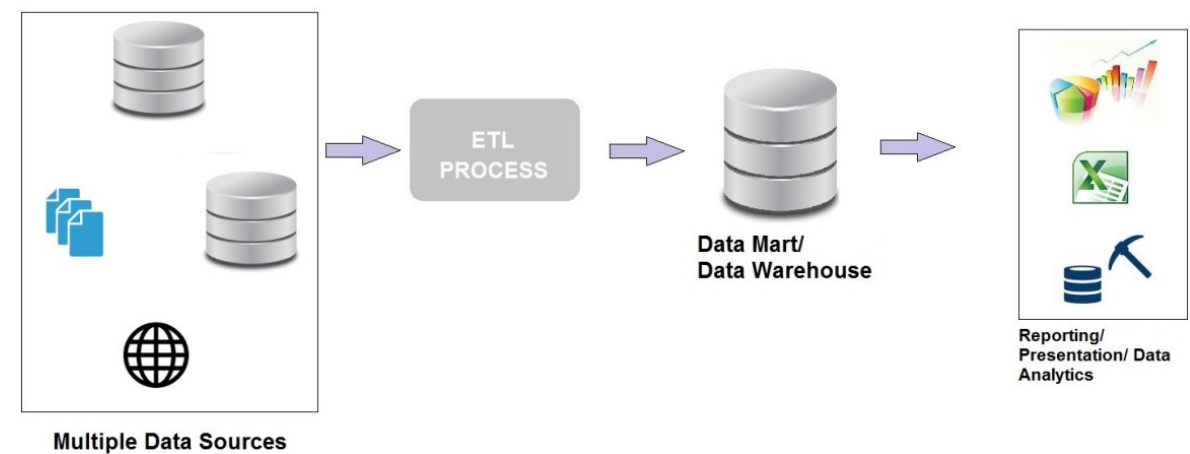

Fig. 1. Key BI components and Processes [26]

In the past, each BI component was developed and supplied by specialist vendors. Selecting the right combination of BI components that would meet the needs of a business requires technical expertise that is often not readily available in the company. The need to integrate various BI components also pushes up the overall implementation cost of a BI solution. In the last decade, with a paradigm shift in the way software components communicate with each other, suppliers have developed, a new generation of BI components have been developed with interoperability in mind and hence could be easily integrated. The cost of implementing BI has therefore been reduced significantly. Some of the major suppliers of BI solutions are well-known names such as Oracle, Microsoft, IBM, SAS and SAP. More recently, BI solutions from smaller specialist vendors such as Qlik and Tableau are gaining traction. To make BI solutions more accessible, some major suppliers of technological solutions such as Microsoft and IBM have begun to supply BI products which cover the entire stack of BI components. Some of these suppliers even deliver BI components as part their standard business solution with no extra cost. For example, a standard suite of Microsoft BI components is included as part of the Microsoft Developer Network (MSDN) license [21]. 
There are a number of open source BI platforms and these have been reviewed and evaluated by Lapa [31] and Ferreira [30]. The BIRT technology Platform [32] allows the creation of data visualizations and reports that can be embedded into other applications, particularly those that have been developed in Java or Java EE. Jaspersoft [33] is a BI solution platform that allows developers to embed reports, dashboards and analytics into an application. Jaspersoft applications can access data from many different database vendor solutions, including SQL Server, Oracle, Mongo DB and MySQL. Jaspersoft solutions can be deployed as Desktop or Mobile applications or accessed as a web application. OpenI [34] is another Java based solution, with its web applications deployed on a Java EE application server. It provides a way to publish reports from OLAP data sources using a Web interface. Jedox [35] is an open source BI solution designed for performance management. It provides functionality for ETL, reporting, planning and analysis. Jedox is designed to integrate with spreadsheet solutions such as Microsoft Excel or Open Office Calc and provides the functionality for the spreadsheet to connect to a Jedox multidimensional database. The Pentaho Data Integration platform [36] provides users with visual tools to simplify data integration. Pentaho can be used to deliver reports, data visualizations and dashboards. Knowage (previously SpagoBI) [37] is an open source solution with functionality for ETL, reporting, OLAP analysis, Microsoft Office Integration, data visualization (including location based visualization) and KPI management. Knowage is a complete BI platform using a modular approach to support different analytical domains. Vanilla [38] is another open source BI platform developed by the French company BPM-Conseil. The Vanilla suite is a set of Java client applications which interact with its application server. Vanilla provides ETL, OLAP analysis, data management and visualization, a metadata tool to create an abstract business layer that is used to create reports and dashboards, and KPI management. Vanilla also provides a plugin to the BIRT platform. While these open source BI solutions include a good range of features to support generation of BI, integrating these BI solutions to a business's IT platform is often not a straight-forward task as it requires a certain level of technical know-how which most SMEs do not readily possess. Furthermore, open source software solutions do not always equate to free software. Hence, the costs for purchasing such software may also be a barrier for BI adoption amongst SMEs.

BI solutions do not come in locally-hosted, server-based form only. Some contemporary BI solutions take the form of Software as a Service (SaaS), which is essentially a cloud-based BI solution. As there is no need for sourcing software and hardware for implementing and hosting the BI solution, the implementation and maintenance costs for SaaS-based BI solutions are minimal. With SaaS, companies simply pay monthly or yearly subscriptions to access the cloud-based service over the Internet. Company data is typically stored in the cloud and managed by the service provider [25]. As new versions of the cloud-based service becoming available, companies using SaaS will be able to utilise the new features instantly. One typical application of SaaS-based BI solutions is in the area of Customer 
Relationship Management (CRM) where the generated intelligence on customer satisfaction is used to improve customer services.

With the availability of inexpensive BI options and a wide range of BI solutions for business use, when adopting BI, what a company needs to do is to identify a suitable strategy to integrate an appropriate BI solution (or components) within the company's existing IT infrastructure. This requires certain level of technical knowhow that may be more readily available in large organisations than small and medium-sized enterprises (SMEs).

\section{$\mathrm{BI}$ in SMEs}

There is a general opinion that SMEs are trailing behind in adopting Business Intelligence to assist in their decision-making. According to a survey conducted by McCabe [16], 33\% of medium-sized companies adopted some kind of BI solutions, and a further $28 \%$ of them planned to take advantage of a BI solution. Amongst smaller organizations, however, only $16 \%$ adopted a BI solution and a further $16 \%$ planned to use a BI solution. While McCabe's survey showed that the adoption of BI amongst SMEs was slow, it also reported that there was an upward trend in the awareness of the need for BI.

The European Commission (EC) defined a small and medium-sized enterprise (SME) as a business with $<250$ staff and a turnover of $\leq € 50 \mathrm{~m}$. About $99 \%$ of businesses in the EU are SMEs [4]. With a relatively small turnover, SMEs typically do not have additional financial and human resources to invest in new, nonbusiness-critical, technologies such BI systems. It therefore comes as no surprise that the adoption of BI amongst SMEs has been slow. Given the cost and complexity involved in adopting a specialist BI solution, many SMEs simply integrate their database with a spreadsheet software such as Microsoft Excel to produce some form of business intelligence [16, 28]. However, as standard spreadsheet software is not equipped with specialist features nor visualization tools to support data analysis and knowledge discovery, the resulting level of intelligence produced is rudimentary and is inadequate to provide a clear view of the company's current performance. Furthermore, for many SMEs, the term Business Intelligence is often misperceived as a technology for large organizations only. This led to a lack of incentive to explore the potential applicability of BI in their businesses. As a result, most decisions made by top level management in SMEs are based on information obtained from various ICT tools built as part of the company's infrastructure [28].

Irrespective of size and sector, there is a general appreciation of the necessity for, and the benefits of, using business intelligence to improve the company's business decision-making process. With the complexity involved in typical BI implementation processes and the lack of appropriate resources, the risk of failure amongst SMEs is high. Hence, many SMEs are put off adopting specialist BI solutions. Moreover, while there are numerous studies reporting successful adoption and utilization of BI amongst large organizations, reports on similar successful 
stories amongst SMEs are uncommon [5]. To promote the uptake of BI solutions amongst SMEs, more needs to be done to raise the awareness of the benefits BI can bring to SMEs and more effort needs to be placed on overcoming the initial challenges faced by SMEs when adopting specialist BI solutions.

\subsection{Benefits of adopting BI in SMEs}

SMEs often operate in a competitive marketplace and under a relatively tight profit margin. To give the business a competitive edge, the management needs to keep abreast of a variety of key business information such as market trend, company performance and its clients' needs. Such information plays an important role in ensuring appropriate company strategies are developed and sound business decisions are made. Specialist BI solutions are designed to generate those kinds of important business intelligence from existing business data.

According to a research by Scholz et al. [27], SMEs can benefit from utilizing BI tools in many ways and the most important ones being: (i) improvements in data support, (ii) improvements in decision support, and (iii) cost and time saving.

In a BI solution, as a data warehouse is designed to facilitate data analysis and retrieval, easy access to business data is therefore guaranteed. The ETL process also ensures that business transaction data is cleansed and validated before entering the data warehouse. Such improvements in data support lead to improved data quality and help ensure the correctness of generated reports.

The visualization and data analytic tools in a BI solution provide rich visuals to help the management better-understand existing business data. Such an improved understanding of the business promotes an accelerated decision-making process. Furthermore, BI tools also help identify risks and hence leading them to be rectified in a timing manner.

One typical feature of BI tools is to generate visual output of business data analysis that are easy to interpret, e.g. in form of dashboards and scorecards. This provides managers and decision-makers with a quick way to identify potential issues within the business.

\subsection{Challenges of BI}

When considering whether or not to adopt a BI solution, SMEs are often confronted with the following issues:

- BI solutions are often expensive. For example, a cloud-based BI solution typically costs at least USD\$500 per month per user. Even with a management team of moderate size, such a monthly cost would add a significant financial burden to the business.

- While off-the-shelf BI tools are available, for non-technical business users, the learning curve of such tools is often too steep to be achievable. 
- Hosting a BI solution requires the support of a non-trivial, and often costly, hardware infrastructure.

- While there is a wide range of BI solutions available, SMEs often lack indepth knowledge of BI to select an appropriate solution for meeting the business's needs.

- Generating BI is often a non-trivial task. It requires advanced knowledge and good understanding of database modelling and data warehousing. Such technical knowledge is often not readily available within most SMEs.

In summary, the challenges can be narrowed down to two main factors: lack of budget and insufficient technical know-how. While these factors pose significant challenges to SMEs in adopting a BI solution, they need not become the barriers to adoption of BI solutions amongst SMEs. To overcome the budget issue, for example, SMEs need to identify low cost BI solutions that will meet their needs. To this end, SMEs may consider adopting an IT solution that comes with a standard BI solution at no extra cost, e.g. Microsoft Office 365 [22]. However, many SMEs may not be aware of such a low-cost BI opportunity nor have the expertise required to start utilizing it. Though to achieve a richer range of data visualization, there may be the need to augment a standard BI solution with data visualization tools. However, the cost of purchasing data visualization tools can be reduced when lightweight web technologies are used to present the results. This, not only minimises the overall cost, it also removes the need for extensive training.

Reflecting on our successful experience with implementing a BI solution for an SME in the UK, in the following sections, we present our adopted approach and discuss how SMEs may overcome the challenges in implementing an effective BI solution.

\section{BI implementation: a case study}

In a competitive business environment, SMEs are increasingly looking for new ways to improve their business decision making process. AGGORA [1], being an SME specializing in providing catering equipment solutions for the food service industry, teamed up with a UK university to create a novel IT platform that aims to deliver new functionality to support their business growth through the use of improved business intelligence. This case study reports the approach adopted in this project.

\subsection{About the Company}

UK based company AGGORA specializes in sales and service of catering equipment. Their clients range from major corporates to small businesses. The company has about 170 staff and has a turnover of approximately $£ 25 \mathrm{~m}$ a year. The market place for AGGORA has become more competitive lately and hence the 
company recognized the need to bring a paradigm shift in their business model, adding IT and servitization expertise to its traditional core strength in equipment and fittings.

AGGORA has grown significantly in recent years and the company plans to continue their rapid growth. To achieve this, the company planned to offer innovative services, extending its range of offers far beyond the traditional design and fabrication, equipment sales, and service and maintenance approach of competitors. They also wanted to have a flexible data management system that will provide valuable performance information on kitchen equipment and fittings from data collected by the company's flagship Asset Management System. Though the inhouse IT systems have the capabilities to generate appropriate information for business analyses and planning, the report generation process was not sufficiently efficient to support the business needs.

AGGORA's leadership team understood the need for radically transforming the business to meet the rapidly changing needs of their marketplace and hence they looked to implement a BI solution to:

a) support their expansion plans,

b) improve their decision making process, and

c) enhance the quality of reports.

The main obstacle which confronted AGGORA was that the company did not possess sufficient technical expertise in BI technologies among its human resource pool and also retraining existing staff was not an option due to time and budget constraints.

To overcome these challenges, AGGORA joined a UK government's scheme called the Knowledge Transfer Partnerships (KTP) scheme. KTP is a UK-wide programme that has been helping businesses for the past 40 years to innovate and grow through access to funding as well as knowledge, technology and skills within UK academic institutions. Each KTP project is a three-way partnership between an SME, an academic institution and a recent graduate. The core aim of all KTP projects is to investigate and implement effective solutions for the identified business challenges. The goal of such a scheme is to transfer research knowledge into SMEs so as to bring about business growth [12]. Every KTP project includes a detailed plan for embedding the knowledge and skills into the company through workshops, training and detailed documentations. This helps ensure maintainability and sustainability of the project on completion.

AGGORA secured the funding for a KTP project and this enabled a graduate to be employed and work on implementing a BI solution with guidance from the partner university. As per the KTP norm, the graduate was employed by the university, but 
was based in the company so as to maximize involvements from all project stakeholders throughout the project development, ensuring that all views and concerns considered and addressed, from the beginning of the project. This helped ensure the design of a BI solution that would address the business needs and a smooth roll out of the BI solution.

\subsection{Project Objectives}

The aims of the KTP project are:

a) to increase the scope of data available for reporting,

b) to minimize the involvement of the IT team by empowering managers to create and maintain their own reports,

c) to create a user-friendly reporting environment, and

d) to lay foundation for a more sophisticated BI solution.

The goal of the project is to implement a suitable BI solution that will empower decision-makers within AGGORA to make more informed business decisions. Figure 2 summarized the goals of the intended BI solution.

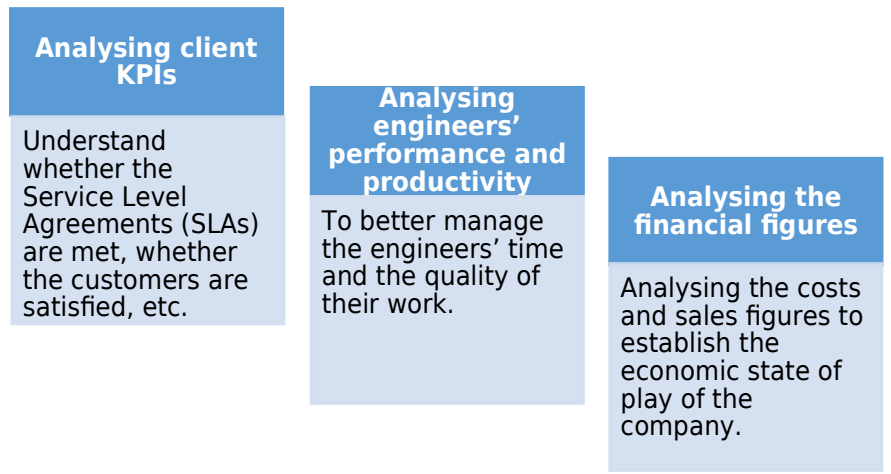

Asset Management

To analyse the reliability of the equipment based on manufacturer and model.

Fig. 2. BI Project Goals. 


\subsection{Implementation Approach}

The implementation approach for a BI solution could significantly vary depending on multiple factors such as the size of the company, budget available for the project and the company's knowledge in implementing a BI solution. Larger organizations with sufficient budget may prefer to buy an off-the-shelf BI solution from a leading vendor. Such solutions would typically offer staff training and support. However, such a solution might be too general and include features that are irrelevant to the organizations. On the other hand, an organization with large IT budget may choose to employ a team of experts to implement and maintain a bespoke BI solution. However, such a solution is unlikely to be affordable and manageable by an SME such as AGGORA.

AGGORA recognizes the need to utilise a BI solution for providing insight into the data from all aspects of its business. However, the company also recognized that implementing a suitable BI solution may not be straight-forward and might have mixed outcomes, including long delays, budget overruns, data problems, and dissatisfied end users.

While trying to accomplish all the requirements and deliver the entire solution all at once may sound ideal, it is unlikely to work well for an SME for the following reasons:

- Changes in business rules and desired metrics may cause delays and conflicts in development.

- There may be the need to resolve legacy data validation issues before the core development commences.

- Requirements could be misinterpreted and hence the deliverables do not meet the requirements.

- There may be significant delays in the development due to changes in business environment and company priorities.

Prompted by constant changes in business priorities and the need for flexibility, we opted for a five staged iterative approach suggested by McGonagle [17] in our BI solution development. Figure 2 shows our adopted approach. The project lasted for 27 months and the BI solution development went through three iterations. 


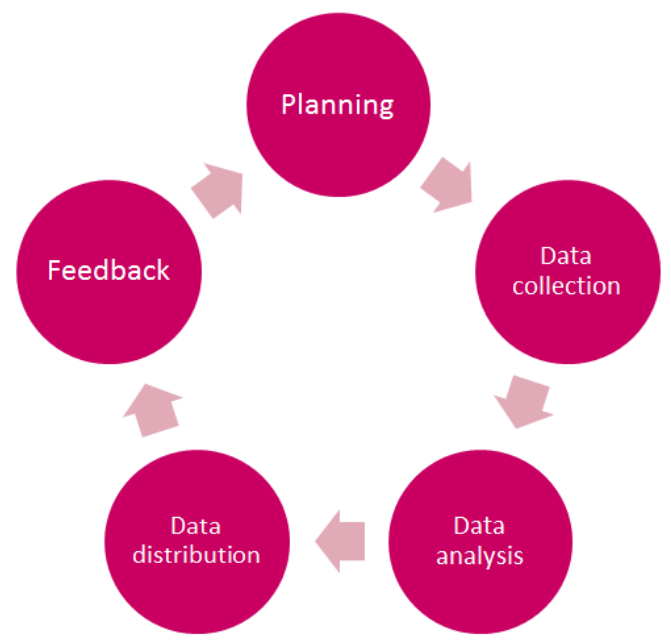

Fig. 3. BI Implementation approach adopted by AGGORA.

Planning. The implementation started with the planning phase. It is a good practice to involve all stakeholders from the management team from the get-go and gather the analysis and reporting requirements up front. In this phase, the management team who would be consuming the intelligence were invited to articulate their requirements and establish the Key Performance Indicators (KPIs) required to be measured. Having all the necessary requirements from the users up front saves a lot of extra rework in the later stages. This also helps to scope out the project and the collected requirements also form the foundation for the subsequent phases.

As there are different tools available for implementing a BI solution, we decided to use the Microsoft suite of BI tools. This decision was prompted by the existing IT infrastructure available in the company. Since the company already uses Microsoft SQL Server 2012 as its backend database along with other Microsoft business products, the Microsoft BI suite facilitated a seamless integration with the company's IT infrastructure. The various components on a Microsoft SQL Server 2012 [20] supported our implementation of the BI solution are:

- SQL Server Database Engine - This includes the Database Engine, the core service for storing, processing and securing data.

- Integration Services - This is a set of graphical tools and programmable objects for moving, copying and transforming data.

- Analysis Services - This includes the tools for creating and managing online analytical processing (OLAP) and data mining applications.

- Reporting Services - This includes server and client components for creating, managing and deploying reports.

- Master Data Services - This is the SQL Server solution for master data management. 
Data Collection. Once the requirements are gathered and objectives are set, we need to transform the data into a format that can be consumed by analytic applications. It was decided that the data from the source would be transformed into and stored in a data warehouse. A data warehouse is fundamentally a database, although there are some significant differences between the design process and best practices for an online transaction processing (OLTP) database and a data warehouse that will support online analytical processing (OLAP) and reporting workloads. With traditional business information systems, the focus is to process business transaction data. Such data is typically stored in a relational database that is designed for facilitating OLTP and is optimized for data entry, retrieval and general transactional processing. With an information system that provides business intelligence, the focus is knowledge discovery and reporting, with online analytic processing being its main task. While these two types of information systems are designed to work with the same data source, their design approaches are very often different due to the need to fulfil different requirements.

One core task in the data collection phase is to identify the business metrics that will drive the data analysis. These metrics are known as the dimensions of the analysis, and the procedure is called dimensional modelling which is a very popular data analytic technique used in the design of data warehouses. The main reasons for its popularity are that it brings about fast query performance and it also presents data in a user consumable format. Although data warehouses can be implemented as normalized relational database schemas, most designs are based on the dimensional model advocated by Ralph Kimball [13]. In a dimensional model, the numeric business measures are stored in fact tables. Each fact table is typically linked to multiple dimension tables that contain the attributes by which the measures can be aggregated.

We have followed Kimball's four steps to dimensional modelling [13]:

- $\quad$ Select the business process.

Business processes are the various operations performed by an organization. Every business process generates events that can be translated into fact tables. Choosing the set of business processes that would be most beneficial for business decision process is paramount. In this project, we have chosen the set of business processes that are relevant to the KPIs we identified in the planning stage.

- Declare the grain.

Declaring the grain is the pivotal step in a dimensional design. The grain defines exactly what should be in a single row of a fact table. The grain should be declared before identifying facts and dimensions to ensure consistency in the design. This consistency is critical for ensuring high performance and ease of use of the resulting BI solution. As a rule of thumb, defining the finest level of grains enables a wider range of business 
intelligence to be generated. Businesses should choose a level of granularity suitable for their requirements.

- Identify the dimensions.

Dimensions specify the "who, what, where, when, why, and how" of business process events. These are essentially the attributes used for filtering and grouping the facts. They contain the descriptive labels that enable the information from a Data Warehouse/Business Intelligence system to be consumed for business analysis. In this project, key parameters such as the customers and the assets for which the performance needs to be measured against were defined as the dimensions.

- Identify the facts.

Facts are the numeric values generated from a business process event. They contain all the measurements needed to provide answers to business questions. Every row in a fact table should be consistent with its corresponding grain. The facts we identified for this project were the numeric measures generated by events in the business process, such as an engineer visiting a client in response to a request to repair equipment.

As proposed by Kimball [13], a data warehouse could be based on two kinds of schemata:

- Star Schema usually consists of fact tables linked to dimension tables using primary/foreign key relationships.

- Snowflake Schema consists of hierarchical relationships in a dimension table, with normalised, low-cardinality attributes appearing as secondary tables connected to the base dimension table by an attribute key.

For the purpose of this project, we have opted for a hybrid approach which is a combination of both star and snowflake schema, partly because we had to integrate with an existing transaction database that was not designed with data warehousing in mind. We have used an iterative approach to build the data warehouse, and in some of the iterations a star schema was more appropriate and in others a snowflake schema was more appropriate. In following such a hybrid approach, we needed to slightly increase the complexity of the resulting data warehouse in order to maintain the data consistency among the shared dimensions. The trade-off for that is a slightly degraded performance although, due to the nature of our data, that slight degradation in performance was estimated to be less than $10 \%$ and not noticeable by end users.

Data Analysis. Once the data warehouse is designed, it has to be populated with data from the live transaction database. This is known as an Extract-Transform-Load (ETL) process.

There are several ways to implement an ETL solution, but SQL Server Integration Services (SSIS) is the primary ETL tool for SQL Server. SSIS includes: 
- SSIS Designer. A graphical design interface for developing SSIS solutions in the Microsoft Visual Studio development environment.

- Wizards. Graphical utilities which enable developers to quickly create, configure, and deploy SSIS solutions.

- Command-line tools. Utilities for managing and executing SSIS packages.

In our project, the source business data is generated from an in-house field service management system and the data is stored in the form of a relational database using Microsoft SQL server. We started by familiarizing ourselves with data source and designed the dimensional modelling for our data warehouse as described above. In order to populate the data warehouse, we implemented an ETL process by using SSIS. This process consisted of three SSIS packages, with each containing one or more Data Flow tasks. The key steps involved in an ETL process are:

\section{a. Extract}

To extract data, an SSIS package must be able to connect to the data source. In an SSIS solution, we defined data connections by creating a connection manager for each data source. As discussed earlier, our source data is the in-house IT system implemented as a single SQL Server database.

\section{b. Transform}

Data transformations enabled us to perform operations on rows of data as they pass through the pipeline. The transformations performed in our ETL process include:

\section{- Row Transformation}

This task deals with the copying of business transaction data to the data warehouse and it is supported by three standard SSIS functions: copy, data conversion and derived column. The Copy Column function adds new columns which are the copies of columns from input data set. The Data Conversion function enables changes to the data type of a column to be made during the translation. The Derived Column function creates new columns derived from the values in the input columns.

\section{- Rowset Transformation}

This task deals with aggregation of atomic data for facilitating data analysis and reporting. The SSIS package provides an Aggregate function which applies aggregation (minimum, maximum, average, sum, etc.) on the incoming set of data

\section{- Split and Join Transformation}

To support data analysis and knowledge discovery, various ways of dividing and/or slicing of data need to take place. A Conditional Split function divides the set of data into more subsets. 


\section{- BI Transformation}

Slowly changing dimension to track the changes and hold the historical information.

\section{c. Load}

Once we have transformed the data into the required format, we load it into a destination data source. In our case, the destination is the data warehouse we designed to hold the pre-aggregated and transformed data.

Data Distribution. The output of the data analysis process is presented to the end users to assist them in making key business decisions. There are various tools available for presenting the information to business users. Microsoft Excel is one of the popular tools used in many organisation for disseminating data but its ability to support a rich presentation of data is limited and it does not provide a good support for data exploration, which is essential for this project as one of our main goals is to enabling self-servicing BI. In this project, we used the cloud-based version of Power BI [18] to present the results of our data analyses to company managers. We chose Power BI over the other self-service BI options available in the market mainly because Power BI is fairly easy to use even for non IT experts and it has the ability to integrate with Office 365 [22]. The skill set required to work with Power BI is very similar to that required to use Excel. With some basic training and documentation, our end users were easily able to generate and deploy reports and dashboards and share them with other users. In order to ensure data integrity, we made sure that the Power BI users can edit their own reports, but not the underlying data.

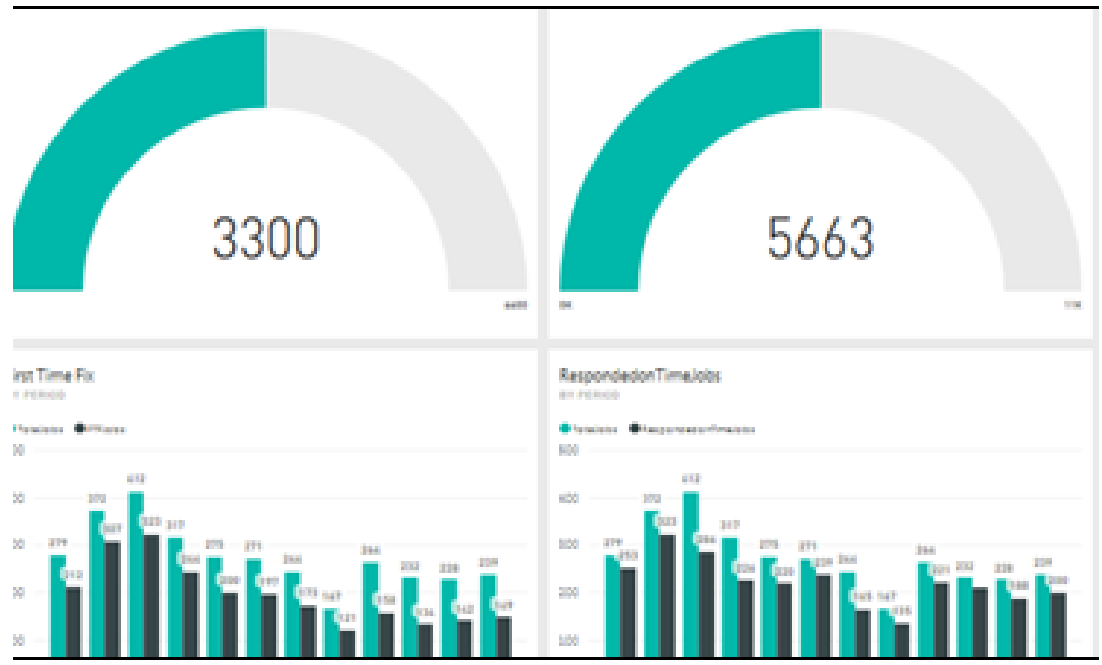

Fig. 4. Sample Power BI Reports 
Though Power BI was used to create and serve ad-hoc reporting requirements, there were some standard reports that should be delivered on a regular basis to the managers. Microsoft SQL Server Reporting Services (SSRS) [23] was used to create and deliver these standard reports. SSRS is a server based report generating software that comes as part of the Microsoft SQL Server suite. The SSRS provides a unique user interface based on Visual Studio that enables developers to connect to the relevant data but unlike Power BI, use of SSRS needed specialist technical knowledge and skills to create and distribute the reports and hence SSRS was not directly available to our end users. In this project, we used SSRS to create some standard KPI reports such as Engineer Performance and Productivity and Breakdown Calls on a monthly basis. These reports are embedded into an email which is sent to the respective managers for their analysis. As these are run-of-themill reports which can be automatically generated once the report generation has been set up, the extra IT staff time required is minimal. Our experience shows that enabling business users to explore the data and generate self-servicing business intelligence is a good approach as this reduces the communication overhead in generating BI reports. Once the business users have established the types of BI reports that are beneficial to their regular business decision-making process, the IT team can then implement these reports using SSRS and have them automatically delivered to the business users on a regular basis.

Feedback. In order for the BI solution to be effective, it is important to generate accurate and high-quality information. As developers we carried out unit testing to ensure our code is free from bugs. However, it was critical for the end users to verify the accuracy of the data and confirm the quality of the reports. Hence, the final step in each implementation iteration was to collect feedback from the end users. This feedback was based on the usability and accuracy of the data that was delivered to them. We made use of this phase to inform the next iteration of implementation. In this project, the feedback we obtained is from a steering group consisting of managers from various levels in the company who are also users of our BI solution. Our steering group members include commercial and technical directors as well as business operation managers.

\subsection{Benefits}

Based on this pilot implementation of a BI solution, we have noticed significant benefits for AGGORA in the first year of implementation. The key beneficiaries have been the top and middle level management. Using Power BI, the management teams have been able to analyse the performance by creating their own on-demand reports without depending on the IT team. This in turn has freed up the time spent by the IT team on creating reports and validating the information. We have estimated the time saving to be around 1.25 days per month. 
The multiple benefits of implementing a BI solution in AGGORA can be classified into three categories:

i. $\quad$ Improvements in data support

This encompasses all the attributes related to reporting and its improvements. With the introduction of Power BI, it has been noted that more reports are being self-serviced than anticipated. The increase in the number of reports is mainly because of the reduction in overall effort involved in reporting and data analysis and the reports are being used in the business management. Power BI being more graphical than Excel has led to improved visualization of the data presented in the reports and also the flexibility to analyse results based on different dimension has accommodated even newer information needs.

ii. Improvements in decision support

This includes all the factors that are associated with better and more informed decision making. The on-demand reports using Power BI and standard reports using SSRS have been precise and present information that was previously not easily accessible, which, which has led to better informed business decisions. Furthermore, the ability to explore a larger subset of data without performance degradation or time lags has led to more timely business decisions being made and also facilitated the identification of business trends and the identification of risks. The use of a specialized data warehouse has also reduced the processing load of the live database, making the core business system more efficient, reliable and effective.

iii. Savings

The time and cost saving achieved by the introduction of the BI solution has been very evident. This implementation has led to a significant amount of time saving in AGGORA's IT department as there is no longer the need for the IT staff to produce reports using queries and pivot tables in an ad hoc manner. This has also opened up more opportunities for the IT staff to widen the scope of information available and improve their efficiency in other business areas.

Table 1 shows the tangible benefits achieved by AGGORA during the project development and the projected tangible benefits to AGGORA one year after the project completion. 
Table 1: Tangible Benefits.

\begin{tabular}{|l|l|r|r|}
\hline & \multicolumn{1}{|c|}{ Tasks } & \multicolumn{1}{|c|}{$\begin{array}{c}\text { Tangible Benefits } \\
\text { Achieved During } \\
\text { Project }\end{array}$} & $\begin{array}{l}\text { Tangibefits } \\
\text { Projected for One } \\
\text { Year After Project } \\
\text { Completion }\end{array}$ \\
\hline 1 & $\begin{array}{l}\text { Increased efficiency of } \\
\text { AGGORA IT team }\end{array}$ & $£ 3,220$ & $£ 5,860$ \\
\hline 2 & $\begin{array}{l}\text { Introduction of PowerBI for } \\
\text { internal reporting }\end{array}$ & $£ 4,202$ & $£ 7,100$ \\
\hline 3 & $\begin{array}{l}\text { New reports built using data } \\
\text { warehouse and PowerBI to } \\
\text { increase efficiency and } \\
\text { revenue }\end{array}$ & $£ 18,060$ & $£ 60,000$ \\
\hline 4 & $\begin{array}{l}\text { Time saving achieved for } \\
\text { IT team by using data } \\
\text { warehouse for generating } \\
\text { report }\end{array}$ & $£ 7,000$ & $£ 13,000$ \\
\hline 5 & $\begin{array}{l}\text { Improved performance of } \\
\text { Asset management system }\end{array}$ & $£ 1,000$ & $£ 1,000$ \\
\hline Total & $£ 33,482$ & $£ 86,960$ \\
\hline
\end{tabular}

\section{Discussion}

In this section, we will summarise our experiences in implementing the first iteration of a BI solution within a UK SME. As a first remark, we understood that it is vital for the BI solution to be user-friendly so that there would be more engagement from non-technical business users allowing them to better understand the benefits of using the solution. In this project, we have tried to abstract the technicality from end users by presenting the analytical results in the form of reports using SSRS and Power BI. The SSRS tool used to generate standard reports requires some technical skills to create and deploy reports. Despite of the technical complexity, the visually rich presentation of the data makes SSRS a powerful dissemination tool. We have noted that as Power BI is very similar to Excel, after some initial basic training, any competent Excel user should be able to use this tool with confidence and without much technical assistance. Power BI supports an intuitive process for creating ondemand reports and it also provides rich data visualisations. Furthermore, up-to-date tutorials demonstrating how to use Microsoft BI tools are readily available, making it highly appealing for end users.

It is important to understand the problem to be solved before implementing a BI solution. A good BI solution should focus on providing answers to important 
business-specific questions. In order for the business users to benefit from those answers, the Key Performance Indicators (KPIs) that will conform the metrics need to be established first. The implementation strategy must be clear and it will depend upon the business environment. In our project, we have used an iterative approach in order to ensure that the project meets the user's changing requirement over time.

As a part of implementation, it is vital to use a well-defined methodology to ensure an efficient BI solution. This requires technical knowledge of core BI concepts such as data warehousing, the ETL process and availability of different data visualisation tools. In terms of delivery strategy, we have chosen to deliver BI in a top-down manner, with the first set of BI solutions rolled out to top-level managers. This allowed top-level management to better understand the benefits of $\mathrm{BI}$, and hence be more supportive of a wider exploitation of BI within the company.

Since BI implementation cannot be a one-off process, the design and implementation should always allow for changes to be made. Such a solution gets embedded within the organisation and starts to work with business. To keep the costs down and to make the solution more accessible to a wide range of end users, we have deliberately used only a limited set of BI tools. The Microsoft suite of BI tools was used for building all aspects of business intelligence required in this project. There are more tools offered in this Microsoft suite of BI application that can be used for more detailed data analytics such as prediction and forecasting.

Finally, our experience shows that:

An effective BI solution lets business users establish the performance metrics and measure their current performance against the KPIs.

While many SMEs understand the benefits of implementing BI solutions, they are often put off by the seemingly complex and expensive implementation process. Our experience shows that affordable and relatively simple BI solutions exist and they can easily be integrated into an SME's existing IT infrastructure.

A thorough understanding of the existing IT infrastructure is necessary to select and implement a custom BI solution within an SME.

Implementing a BI solution is a non-trivial process and involves several phases. A good understanding of these phases is needed to overcome some of the technical challenges. We have found that there is no need for training the entire team of IT staff up-front. Training up a single recent IT graduate is sufficient to gradually roll out the development process.

In order to make the BI solution successful, accurate intelligence needs to be delivered. It is important to have high-quality data which can achieved by identifying the data problems early and transforming and cleansing the data during the ETL process. 
It is vital to use a well-defined methodology to design the data warehouse. In our project, we have used dimensional modelling approach to ensure efficient data retrieval and analysis.

The Microsoft suite of BI tools provides all the required components to implement an enterprise wide BI solution. This makes them perfectly suitable for SMEs wanting to implement BI solutions, who are already using other Microsoft business products as a part of their IT infrastructure.

While technical barriers do exist, there are government schemes, in the case of UK and Europe, available to help SMEs to overcome such barriers through funding, recruitment and knowledge transfer.

This paper describes our first step in implementing a BI solution to an SME in the UK. Our next step includes using BI to: (1) achieve further potential enhancements within the organisation, (2) analyse the productivity of sub-teams within the enterprise and (3) perform margin analysis or implementing a more sophisticated account statement model.

With the option available to integrate R scripts with Power BI, we also plan to investigate using predictive time series analysis or other advanced data analysis to provide forecasts.

\section{Conclusion}

BI tools provide analytic data and key performance information which enables organisations of all sizes to be managed efficiently. It helps organisations to overcome the challenges involved in knowledge management and discovery. An efficient BI solution would potentially reduce the cost spent on resource and time to extract intelligence from the available data. It materializes the management's vision by empowering them with the ability to make more informed business decisions by minimizing the error on even large and complex data sample.

Based on our experience, we have understood that it is importance for SMEs to streamline their information resource in order to make more informed business decisions. We were able to appreciate the challenges that an SME could encounter while trying to implement a BI solution. In general, SMEs may not possess sufficient technical expertise that is needed to implement and maintain a custom BI solution. Furthermore, choosing the best solution from a densely populated analytics market is challenging and adds to the complexity.

In this case study, we have elaborated our approach to implement a BI solution for a UK based SME. Due to the volatile nature of the business within AGGORA, we opted for an iterative approach to implement the BI solution. It is important to 
establish the KPIs to lay the foundation for the implementation of the project. Considering the existing IT infrastructure, we decided to use Microsoft suite of BI tools to implement the BI solution as that presented a shallower learning curve for the end users and enabled early benefits to the company.

We have also highlighted the need for using a sophisticated design approach to model the data warehouse. We have used the Kimball's approach for designing our data warehouse in order to ensure efficient and fast data retrieval. The source data was transformed and cleansed using the Microsoft integration services. The transformed data is presented to business users using a range of data presentation tools.

One of the main objectives of this project was to empower the business users with all relevant information to make more informed business decisions. We have delivered business intelligence using self-service BI and also standard reports. Selfservice BI delivered using Power BI empowers managers to explore the available data and generate their own reports and dashboards without any involvement from the IT team. This has made the IT team more efficient, leading to cost saving from no longer having to generate ad-hoc reports. The standard reports delivered on a regular basis using Microsoft reporting services helped the management team to identify patterns in performance transaction data which leads to improvements in the efficiency and productivity of the company and better informed management decision making.

We appreciate the possible limitations of our work. As this case study is based on a company which has already adopted a Microsoft-based IT infrastructure, we are mindful that the costs, benefits, implementation approach and time scales could be different for companies using different IT products within their infrastructure. However, with Microsoft being the market leader in both operational database management systems and BI and analytics platforms [7, 8], our findings are relevant to a large number of SMEs.

This paper describes our first step in implementing a BI solution to an SME in the UK. Our next step includes using BI to: (1) achieve further potential enhancements within the organisation, (2) analyse the productivity of sub-teams within the enterprise and (3) perform margin analysis or implementing a more sophisticated account statement model.

With the option available to integrate R scripts with Power BI, we also plan to investigate using predictive time series analysis or other advanced data analysis to provide forecasts.

Acknowledgements. The project reported in this case study is funded by Innovate UK via the Knowledge Transfer Partnership Scheme (KTP009714). We acknowledge the financial support by Innovate UK. We also acknowledge the 
advice provided by our KTP Adviser Ms Jose Freeman throughout the project development. We thank the team at AGGORA who made this project possible through their participation.

\section{References}

1. AGGORA Group. (2016). AGGORA, http://aggora.co.uk/ [accessed 8 March 2017].

2. Burstein, F. and Holsapple, C. (2008). Handbook on decision support systems. Berlin: Springer.

3. Chugh, R and Grandhi, S. (2013). Why Business Intelligence? Significance of Business Intelligence tools and integrating BI governance with corporate governance', International Journal of

E-Entrepreneurship and Innovation, vol. 4, no.2, pp. 1-14. http://www.igiglobal.com/article/why-business-intelligence/89282

4. European Commission (2016). What is an SME?, http://ec.europa.eu/growth/smes/business-friendly-environment/smedefinition/index en.htm [accessed 8 March 2017].

5. Fink, K. and Ploder, C. (2009). Knowledge Management Toolkit for SMEs. International Journal of Knowledge Management, 5(1), pp.46-60.

6. Gartner Inc. (2012). Gartner Executive Programs' Worldwide Survey of More Than 2,300 CIOs Shows Flat IT Budgets in 2012, but IT Organizations Must Deliver on Multiple Priorities, http://www.gartner. com/newsroom/id/1897514 [accessed 07 March 2017].

7. Gartner Inc. (2015). Magic Quadrant for Operational Database Management

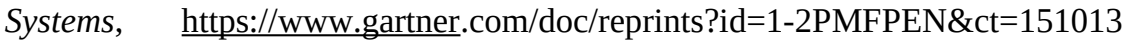
[accessed 15 August 2016].

8. Gartner Inc. (2016). Magic Quadrant for Business Intelligence and Analytics Platforms, https://www.gartner.com/doc/reprints?id=1-2XXET8P\&ct=160204 [accessed 15 August 2016].

9. Google (2016). Google Charts, https://developers.google.com/chart/ [accessed 8 March 2017].

10. Horakova, M. and Skalska, H. (2013). Business Intelligence and Implementation in a Small Enterprise. Journal of Systems Integration, 4.2, pp.50-61.

11. Howson, C. (2008). Successful business intelligence. New York: McGraw-Hill.

12. InnovateUK (2016), Knowledge Transfer Partnerships, https://connect.innovateuk.org/web/ktp [accessed 7 March 2017].

13. Kimball, R. and Ross, M. (2013). The Data Warehouse Toolkit: The Definitive Guide to Dimensional Modeling, $3^{\text {rd }}$ edition. Indianapolis, IN: Wiley.

14. Lacko, L. (2009). Business Intelligence v SQL Serveru 2008. Brno: Computer Press. 
15. Luhn, H.P. (1958). A Business Intelligence System, IBM Journal of Research and Development, 2(4), 314-319.

16. McCabe, L. (2012). Closing the Business Intelligence Gap for Small Businesses, https://lauriemccabe.com/2012/ 01/27/closing-the-businessintelligence-gap-for-small-businesses/ [accessed 8 March 2017].

17. McGonagle, J. (2007). An Examination of the ' Classic ' CI Model. Journal of Competitive Intelligence and Management, 4(2), pp.71-86.

18. Microsoft. (2015). Frequently asked questions about Power BI Microsoft Power BI, https://powerbi. microsoft.com/en-us/documentation/powerbifrequently-asked-questions/?CorrelationId=a0b3a797-a620-4e09-b3461141a5f1d03d\&ui=en-US\&rs=en-US\&ad=US [accessed 8 March 2017].

19. Microsoft. (2016a). DAX Queries, https://msdn.microsoft. com/enus/library/gg492201.aspx [accessed 7 March 2017].

20. Microsoft. (2016b). Editions and Components of SQL Server 2012, https://technet.microsoft.com/en-us/libra $\mathrm{ry} / \mathrm{ms} 144275 \% 28 \mathrm{v}=\mathrm{sql} .110 \% 29 . \mathrm{aspx}$ [accessed 8 March 2017].

21. Microsoft. (2016c). Microsoft Developer Network (MSDN), https://msdn.microsoft.com/ [accessed 8 March 2017].

22. Microsoft. (2016d). Office 365, https://products.office.com/engb/business/explore-office-365-for-business [accessed 8 March 2017].

23. Microsoft. (2016e). Reporting Services (SSRS), https://msdn.microsoft.com/enus/library/ms159106.aspx [accessed 8 March 2017].

24. Negash, S. and Gray, P. (2003) Business intelligence, Proceedings of the Ninth Americas Conference on Information Systems (AMCIS 2003), Tama, Florida, pp. 3190-3199.

25. Papazoglou, M. P. (2003). Service-oriented computing: concepts, characteristics and directions, Proceedings of the Fourth International Conference on Web Information Systems Engineering, 2003 (WISE 2003), Rome, pp. 3-12. doi: 10.1109/WISE.2003.1254461.

26. Raj, R., Wong, S.H.S. and Beaumont, A.J. (2016). Business Intelligence Solution for an SME: A Case Study. Proceedings of the 8th International Joint Conference on Knowledge Discovery, Knowledge Engineering and Knowledge Management (IC3K 2016) - Volume 3: KMIS, pp. 41-50. SCITEPRESS -Science and Technology Publications, Lda. ISBN: 978-989-758-203-5.

27. Scholz, P., Schieder, C., Kurze, C., Gluchowski, P. and Böhringer, M. (2010). Benefits and challenges of business intelligence adoption in small and mediumsized enterprises. 18th European Conference on Information Systems.

28. Tutunea, M.F. and Rus, R.V. (2012). Business intelligence solutions for SME's. Procedia Economics and Finance, 3, pp.865-870.

29. Watson, H. and Wixom, B. (2007). The Current State of Business Intelligence, Computer, 40(9), pp.96-99.

30. Ferreira, T., Pedrosa, I. and Bernardino, J. (2017) Evaluating Open Source Business Intelligence Tools using OSSpal 
Methodology. In Proceedings of the $9^{\text {th }}$ International Joint Conference on Knowledge Discovery, Knowledge Engineering and Knowledge Management (KDIR 2017). pp.283-288.

31. Lapa, J., Bernardino, J. and Figueiredo, A. (2014). A comparative analysis of open source business intelligence platforms. In Proceedings of the International Conference on Information Systems and Design of Communication (ISDOC '14). ACM, New York, NY, USA, pp.86-92.

32. The Eclipse Foundation. (2014). BIRT, http://www.eclipse.org/birt/ [access 29 April 2018].

33. TIBCO Software. (2018). TIBCO Jaspersoft, https://www.jaspersoft.com/ [access 29 April 2018].

34. OpenI.Org. (2008). OpenI: Open Source Web Application for OLAP Reporting, http://openi.sourceforge.net/openi product.html [access 29 April 2018].

35. Schmeider, H., Jakl, M. and Haddadin, K., (2015). Jedox, https://sourceforge.net/projects/palo/ [access 29 April 2018].

36. Hitachi Vantara. (2018). Pentaho Data Integration, https://www.hitachivantara.com/en-us/products/big-data-integrationanalytics/pentaho-data-integration.html [access 29 April 2018].

37. Engineering Ingegneria Informatica S.P.A. (2018). Knowage, https://www.spagobi.org/ [access 29 April 2018].

38. BPM-Conseil. (2018). Vanilla - Analytics Business Intelligence for Big Data, http://www.bpm-conseil.com/ [access 29 April 2018]. 